\title{
Two-Stage Assembly of Mesocrystal Fibers with Tunable Diameters in Weak Magnetic Fields
}

\author{
Martin Kapuscinski,* Pierre Munier, Mo Segad, and Lennart Bergström* \\ Cite This: Nano Lett. 2020, 20, 7359-7366 \\ Read Online
}

ABSTRACT: Controlling the morphology and crystallographic coherence of assemblies of magnetic nanoparticles is a promising route to functional materials. Time-resolved small-angle X-ray scattering (SAXS) was combined with microscopy and scaling analysis to probe and analyze evaporation-induced assembly in levitating drops and thin films of superparamagnetic iron oxide nanocubes in weak magnetic fields. We show that assembly of micrometer-sized mesocrystals with a cubic shape preceded the formation of fibers with a high degree of crystallographic coherence and tunable diameters. The second-stage assembly of aligned cuboidal mesocrystals into fibers was driven by the magnetic field, but the first-stage assembly of the oleate-capped nanocubes was unaffected by weak magnetic fields. The transition

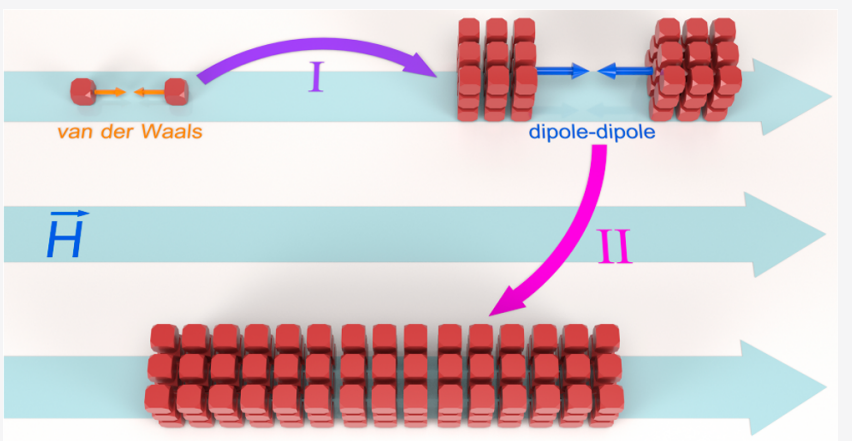
from 3D growth of the primary mesocrystals to the second stage $1 \mathrm{D}$ assembly of the elongated fibers was related to the size and field dependence of isotropic van der Waals and directional dipolar interactions between the interacting mesocrystals.

KEYWORDS: self-assembly, magnetic field, SAXS, mesocrystal, iron oxide, nanoparticle

$\mathrm{D}$ irectional assembly of superparamagnetic nanoparticles in external magnetic fields has been used to generate assemblies and mesocrystals ${ }^{1-3}$ with potential applications in biomedicine and for hyperthermia ${ }^{4-6}$ or optoelectronics. ${ }^{7}$ The magnetic properties of the assembled materials depend on the size, degree of order, and in particular the morphology of the anisotropic field-directed assemblies. ${ }^{8-11}$ One-dimensional chain-like assemblies often display enhanced magnetization along the long axis. ${ }^{12-15}$

The morphology and structure of nanoparticle arrays formed by the assembly of anisotropic superparamagnetic iron oxide nanoparticles (with sizes $\leq 20-30 \mathrm{~nm}$ ) in moderate-to-strong magnetic fields has been studied primarily by ex situ techniques. Wetterskog et al. used electron microscopy (EM) and ex situ grazing incidence small-angle X-ray scattering (GISAXS) to show that the strength of a magnetic field, oriented perpendicular to the substrate, modulated the morphology of evaporation-induced self-assembled superparamagnetic iron oxide nanocube mesocrystals, where single domain, multidomain, and aligned mesocrystals were formed in zero field, $65 \mathrm{mT}$, and $200 \mathrm{mT}$ magnetic fields, respectively. ${ }^{16}$ Polyhedral iron oxide nanoparticles assembled at moderate-to-strong magnetic fields $(15-200 \mathrm{mT})$ parallel to a substrate surface or liquid-liquid interface resulted in elongated chain-like structures. ${ }^{17,18}$ Klajn and co-workers combined electron microscopy and modeling to show that assemblies of superparamagnetic nanocubes formed at moderate field strengths $(17 \mathrm{mT})$, where the magnetic moments aligned in a zigzag pattern, whereas assembly at higher field strength $(67 \mathrm{mT})$ resulted in thinner chains and eventually in the formation of helical structures due to competition of magnetic and spatial symmetries. ${ }^{19}$ In situ studies are rare, but snapshots at different stages of fieldinduced assembly of superparamagnetic nanoparticles by smallangle neutron scattering could be related to the formation of one-dimensional chains and 3D superlattices at moderate magnetic fields $(\geq 10-20 \mathrm{mT}) .^{20}$ Assembly studies performed in thin films or at interfaces have shown that assembly of superparamagnetic nanoparticles at relatively strong magnetic fields usually results in chain-like morphologies, ${ }^{17-19,21-23}$ whereas evaporation-induced assembly in the absence of magnetic fields results in the formation of large 3D assemblies. The distinctly different morphologies suggest that transitions from $3 \mathrm{D}$ to $\mathrm{ID}$ assembly could occur in weak magnetic fields where the magnitude of the directional dipolar interaction and isotropic vdW interaction ${ }^{24}$ are of similar magnitude. Clarifying the details of the $3 \mathrm{D}$ to $1 \mathrm{D}$ transition can give important insights to how to control the morphology of assemblies of

Received: July 3, 2020

Revised: September 8, 2020

Published: September 14, 2020 

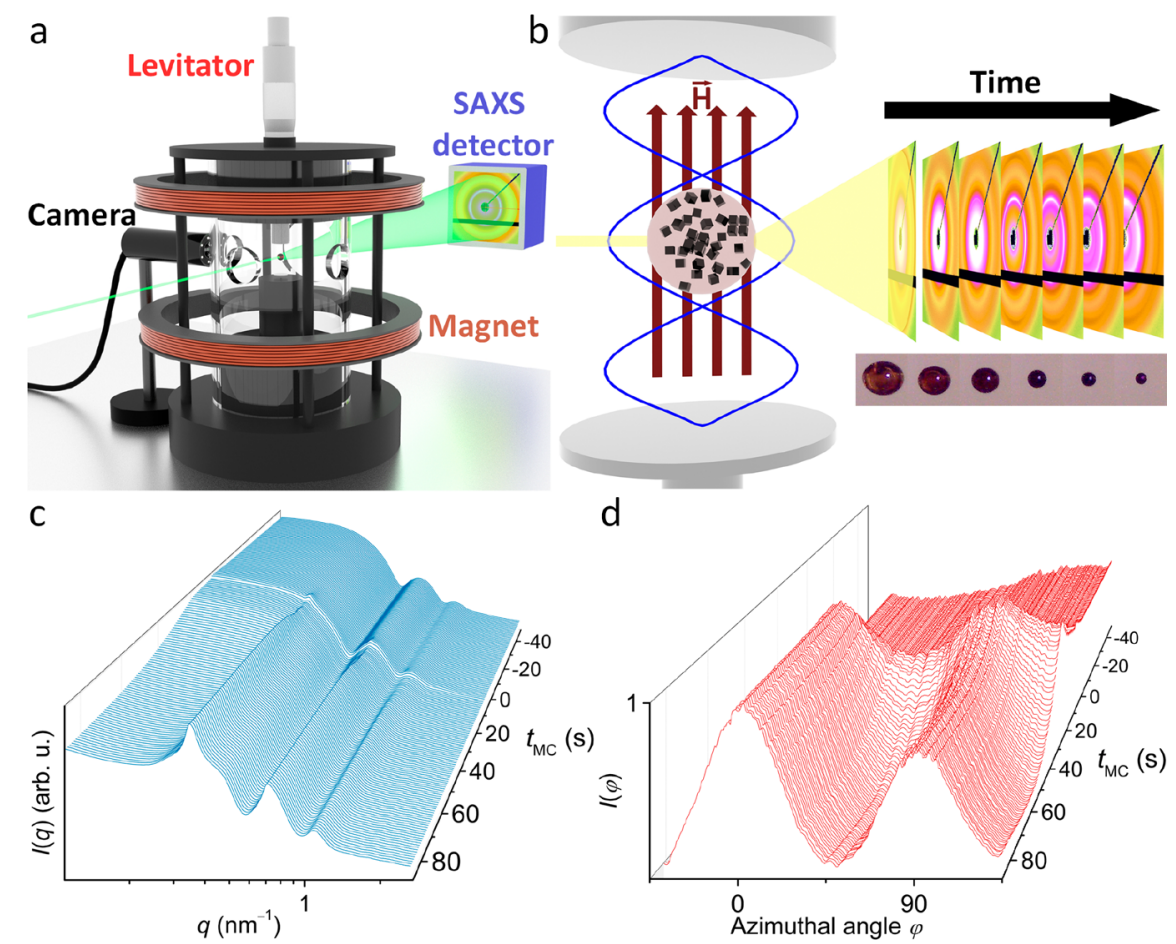

Figure 1. Experimental setup, as well as time-resolved video and SAXS data. (a) Schematic illustration of the experimental setup used for the timeresolved SAXS experiments. The acoustic levitator is equipped with a custom-built chamber with ports for ingoing and scattered X-ray beams, a microscope camera, and sample injection. The Helmholtz coil produced a magnetic field of $1.5-6 \mathrm{mT}$. (b) The vertical magnetic field (H) acts on the levitating colloidal droplet. The size of the shrinking droplet and 2D SAXS patterns were collected throughout the assembly process. Excerpts of (c) radially and (d) azimuthally integrated, time-resolved SAXS curves of a dispersion droplet containing NC120.

superparamagentic nanoparticles, which can be of relevance to magnetorheological fluids and assembly of spintronic components. $^{25,26}$

We conducted real-time small-angle X-ray scattering (SAXS) measurements in a levitating droplet (Figure 1a), which, in contrast to thin films, offers an unconfined medium for selfassembly, and complemented these measurements with optical microscopy to probe the magnetic-field-directed assembly of oleate-capped superparamagnetic magnetite nanocubes (NCs). We show that the self-assembly of $12 \mathrm{~nm}$ magnetite nanocubes in a weak magnetic field proceeds in two stages. The first stage is dominated by the assembly of the magnetite NCs into micrometer-sized mesocrystals with a cubic morphology. These "primary" mesocrystals then assemble into elongated mesocrystalline fibers with a high degree of positional order and crystallographic coherence. We have estimated the van der Waals and induced dipolar interactions and will discuss how the relative importance for the oriented attachment and assembly processes varies during mesocrystal growth. The possibility to control the magnitude of the magnetic field allows for the fabrication of ordered assemblies of magnetic nanoparticles with tunable morphology.

The assembly was performed in a homogeneous magnetic field as the levitating droplet was irradiated with an X-ray beam (Figure 1a). A droplet with an initial volume of $2-3 \mu \mathrm{L}$ and a concentration of $3 \mathrm{mg} \mathrm{mL}^{-1}$ magnetite NCs levitated between two pressure nodes of the standing acoustic wave (Figure $1 \mathrm{~b}$ ). As the solvent evaporated, the shrinking droplet was observed with a microscope camera, allowing the droplet volume to be measured and the average $\mathrm{NC}$ concentration to be estimated as a function of time (Figure $1 b$ ). The time-resolved SAXS studies were performed in a 3:1 toluene/decane mixture, in which the evaporation rate is sufficiently slow to allow data to be collected with high time resolution. Comparative experiments in pure toluene and in toluene/decane mixtures showed that the solvent mixture does not influence the structure of the final mesocrystals.

The morphology and size of the NCs and the structure of the mesocrystals were obtained by radially integrating the timeresolved 2D SAXS patterns (Figure 1c). The average edge length of the single crystalline NCs, $12.0 \pm 0.8 \mathrm{~nm}$ (NC120), was obtained by fitting the SAXS curves of dilute dispersions to a cubic form factor (Figure S1). Indexing the structural peaks that appeared as the droplet shrank and the particle concentration increased showed that the NCs assembled face-to-face into a simple cubic (SC) lattice (Figure S2).

Azimuthal integration of the 100 diffraction ring in the $2 \mathrm{D}$ SAXS image of a levitating droplet containing NC120 nanocubes (Figure 1d) showed pronounced peaks at azimuthal angles $(\varphi)$ of $0^{\circ}$ and $90^{\circ}$, which correspond to the alignment of the $\{100\}$ mesocrystal planes with respect to the magnetic field. The $90^{\circ}$ intervals of the azimuthal peaks suggests that the mesocrystals have a 4-fold symmetry, corroborating the simple cubic structure determined by indexing the scattering pattern of the mesocrystals (Figure S2).

The time-dependent partial scattering invariant $Q^{*}\left(t-t_{\mathrm{MC}}\right)$ $\left.\propto \phi_{\mathrm{NC}}\left(1-\phi_{\mathrm{NC}}\right)(\Delta \rho)^{2}\right)$, which is related to the scattering length density contrast $\Delta \rho$ between iron oxide and the solvent, as well as to the volume fraction of the $\mathrm{NCs} \phi_{\mathrm{NC}}$, was obtained by

$$
Q^{*}=\int_{q \min }^{q \max } I(q) q^{2} \mathrm{~d} q
$$




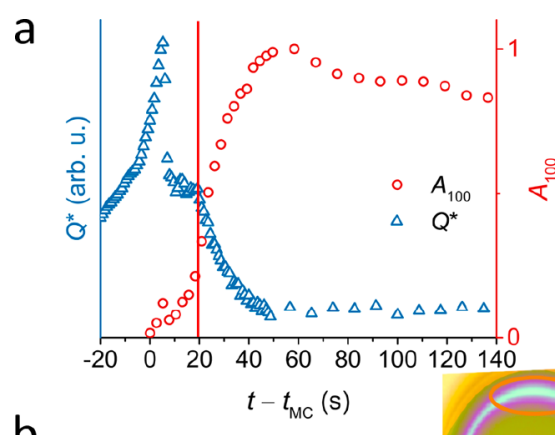

b

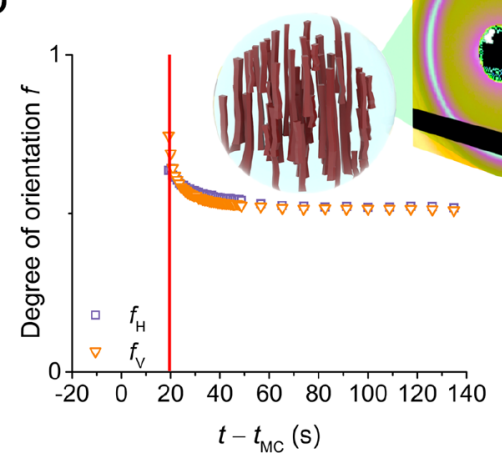

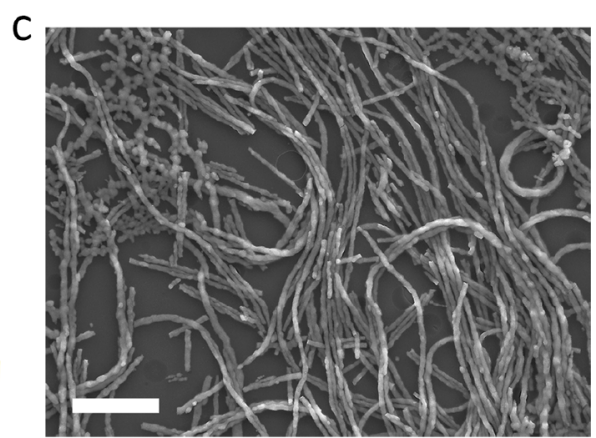

d

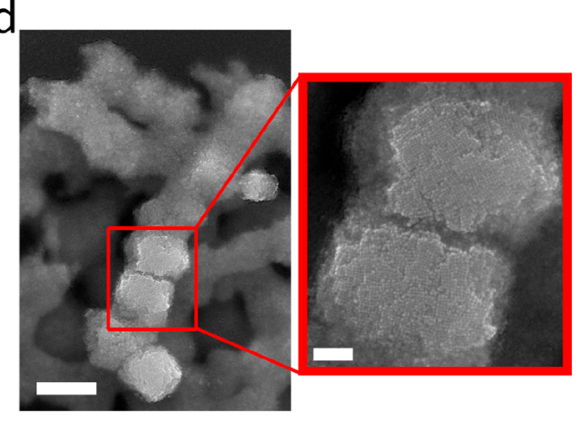

Figure 2. Temporal evolution of $A_{100}, Q^{*}$, and $f$ and the morphology of mesocrystals grown by self-assembly of NC120 in a $6 \mathrm{mT}$ magnetic field. (a) Normalized peak area $A_{100}$ of the 100 superlattice peak (red circles) and partial scattering invariant $Q^{*}$ (blue triangles). (b) Degree of orientation $f$ in the horizontal (purple squares) and vertical (orange triangles) direction. The red vertical line depicts the end of the plateau region (a) and the first appearance of alignment (b). The inset displays a schematic illustration of the 2D diffraction image and the aligned mesocrystal. The vertical (orange ellipse) and horizontal (purple ellipse) directions are highlighted. Scanning electron microscopy (SEM) images of (c) the mesocrystalline fibers (scale bar $=10 \mu \mathrm{m}$ ) and $(\mathrm{d})$ of a shorter fiber (scale bar $=1 \mu \mathrm{m}$ ) that consists of small, cuboidal mesocrystals (inset; scale bar $=200 \mathrm{~nm})$.
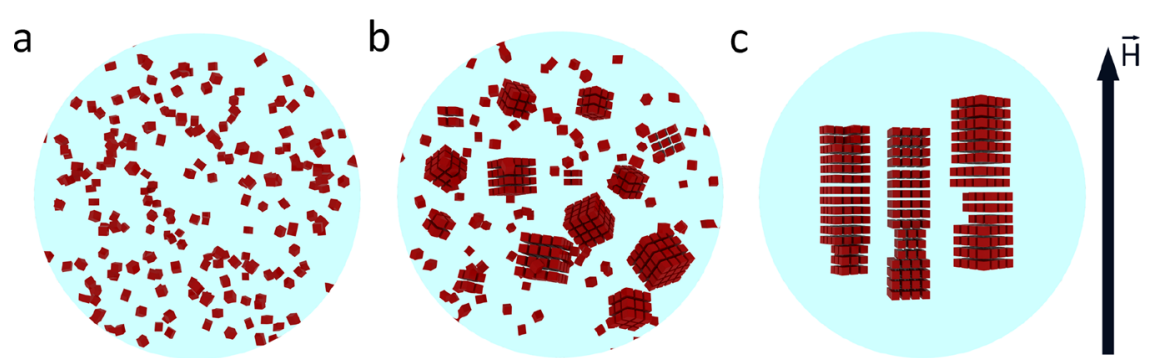

Figure 3. Schematic illustration of the two-stage assembly of iron oxide nanocubes (NCs) in a magnetic field $\vec{H}$ (blue arrow). (a) The welldispersed NCs; (b) evaporation-driven assembly of the NCs generate the primary cuboidal mesocrystals; and (c) the cuboidal mesocrystals align and attach in an oriented fashion along the magnetic field to form mesocrystal fibers.

with $I(q)$ being the measured intensity over the range $0.11<q$ $<2.64 \mathrm{~nm}^{-1}$. The partial scattering invariant was related to the time $t-t_{\mathrm{MC}}$, with $t$ being the experimental time (which relates to when the first scattering pattern was obtained), and $t_{\mathrm{MC}}$ the time when the mesocrystals first appeared, indicated by the appearance of structural peaks (Figure 2a). ${ }^{27-29}$ The invariant $Q^{*}\left(t-t_{\mathrm{MC}}\right)$ displayed a maximum about $5 \mathrm{~s}$ after the beginning of crystallization and then decreased, first rapidly and eventually more gradually until a plateau was reached that ended at $t-t_{\mathrm{MC}}=19 \mathrm{~s}$ (marked by a red line). $A_{100}$ increased rapidly between 19 and $50 \mathrm{~s}$, where it reached a maximum that coincided with $Q^{*}$ reaching a steady-state value.

The rapid reduction of $Q^{*}\left(t-t_{\mathrm{MC}}\right)$ for $t_{\mathrm{MC}}>5 \mathrm{~s}$ suggests that the density of X-ray scattering objects with sizes $2.4-57$ $\mathrm{nm}$, corresponding to the evaluated $q$-range, is reduced. The average nanocube volume fraction increased by $170 \%$ during the investigated period $\left(t_{\mathrm{MC}}-20 \mathrm{~s}\right.$ to $\left.t_{\mathrm{MC}}+100 \mathrm{~s}\right)$, which is expected to increase $Q^{*}\left(t-t_{\mathrm{MC}}\right)$ by $150 \%$. The observed reduction of $Q^{*}\left(t-t_{\mathrm{MC}}\right)$ could indicate a migration of the growing mesocrystals to regions outside the $\mathrm{X}$-ray beam, e.g., the air-liquid interface, ${ }^{29}$ or that the growing mesocrystals became so large that parts of the scattering pattern fell outside the probed $q$-range. ${ }^{30,31}$ The plateau period of $Q^{*}\left(t-t_{\mathrm{MC}}\right)$ at $t$ $-t_{\mathrm{MC}}=10-19 \mathrm{~s}$ suggests that the amount or size of nanoparticles/mesocrystals remained relatively constant, which may indicate the end of the first mesocrystal growth stage.

As $A_{100}$ increases rapidly and $Q^{*}$ decreases, a pronounced alignment is visible in the azimuthal plot of the SAXS data. The degree of mesocrystal orientation in the horizontal and vertical directions $\left(f_{\mathrm{H}}\right.$ and $f_{\mathrm{V}}=\left(90^{\circ}-\right.$ fwhm $) / 90^{\circ}$; fwhm $=$ full-width at half-maximum of the Gaussian peaks at $0^{\circ}$ and $90^{\circ}$ in the azimuthal plot, Figure $2 \mathrm{~b}$ ) is highest at $t-t_{\mathrm{MC}}=19 \mathrm{~s}$, then decreases slightly and reaches steady state at about $t-t_{\mathrm{MC}}=$ $50 \mathrm{~s}$. The maximum degree of alignment of the nanocubes at $t$ $-t_{\mathrm{MC}} \sim 20 \mathrm{~s}$ suggests that alignment of the cuboidal mesocrystals in the magnetic field precedes the initiation of the 


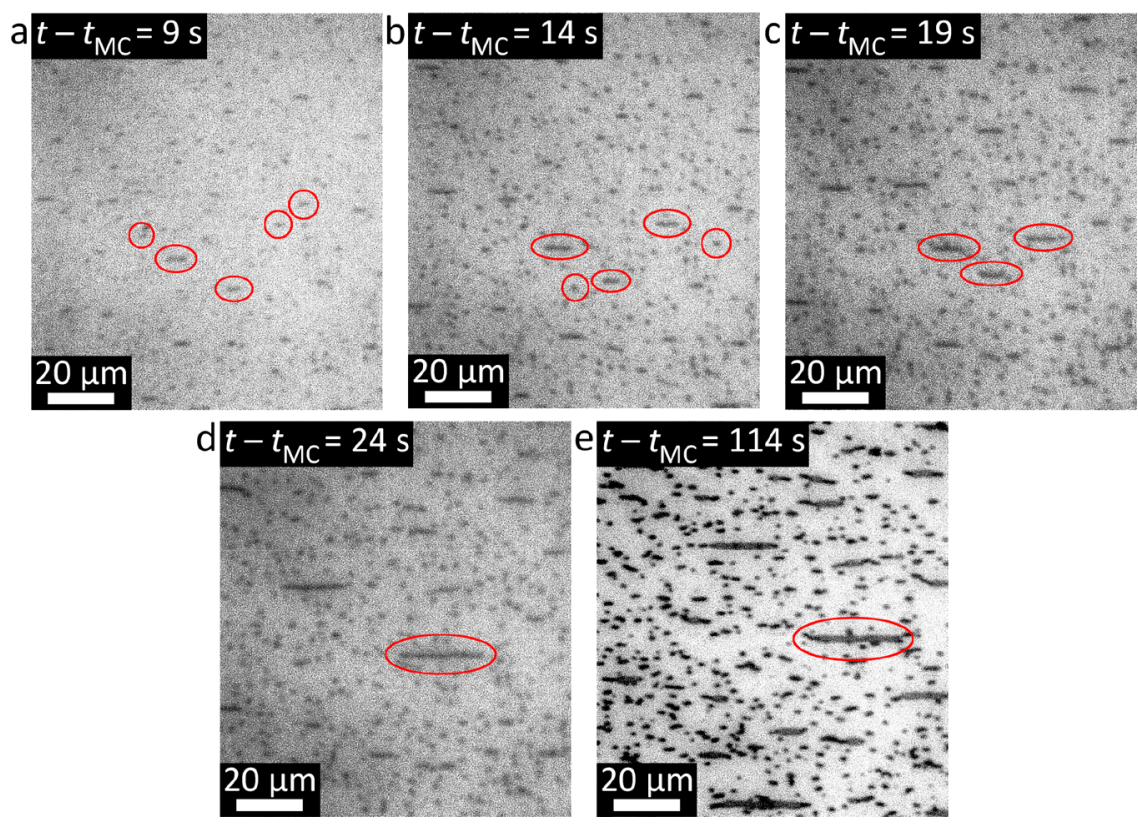

Figure 4. Formation of elongated fibers by self-assembly of NC120 in a magnetic field of $6 \mathrm{mT}$. Primary cuboidal mesocrystals attach and form mesocrystalline fibers (red ellipses) whose length increases with time. The magnetic field is horizontally oriented. The growth of the fibers is illustrated by snapshots of a single region at different times $t-t_{\mathrm{MC}}$ after mesocrystal formation was observed: (a) $9 \mathrm{~s}$, (b) $14 \mathrm{~s}$, (c) $19 \mathrm{~s}$, (d) $24 \mathrm{~s}$, and (e) $114 \mathrm{~s}$.

second assembly stage. The subsequent reduction of the degree of alignment during the second assembly stage may be related to misalignments of the attached mesocrystals during the rapid growth of the fibers.

The dried beads that were collected after the SAXS experiment contained predominantly mesocrystalline fibers, which indicates that the yield of the second-stage assembly process was high (Figure 2c). The fibers consisted of smaller cuboidal, well-ordered mesocrystals (Figure 2d), which suggests that the fibers formed by the assembly and alignment of the cuboidal "primary" mesocrystals. Estimates of the diffusion length for NC120 (see SI) suggests that the assembly of nanocubes into cuboidal mesocrystals is not diffusion limited but is probably controlled by the slower process of aligning and attaching the nanocubes in a low-energy configuration into the growing mesocrystal. ${ }^{32,33}$

Figure 3 shows a schematic illustration of the two-stage assembly process where the evaporation-driven assembly of the dispersed nanocubes (Figure 3a) generates the primary mesocrystals (Figure $3 \mathrm{~b}$ ) that are assembled and aligned by the magnetic field to form fibers (Figure 3c). The primary mesocrystals, grown in the presence of a magnetic field $\left(\mathrm{MC}_{\mathrm{NC120,MF}}\right)$, displayed the same crystal structure (Figure $\mathrm{S} 2$ ) and critical volume fraction (Figure $\mathrm{S} 3$ ) as the mesocrystals grown in the absence of a field $\left(\mathrm{MC}_{\mathrm{NC120,MF}}\right)$, which shows that the weak magnetic field has a negligible effect on the primary growth stage. Mesocrystalline fibers only formed when the dispersion was subjected to a weak magnetic field, demonstrating that the magnetic field directed the assembly and alignment during the secondary growth stage. Confinement effects within the levitating droplets are expected to be insignificant because the droplet diameters that range from 1500 down to around $300 \mu \mathrm{m}$ for the nearly dry bead are more than 100 times larger than the cuboidal mesocrystals and several times larger than the lengths of the mesocrystalline fibers. The predominant linear morphology of the fibers supports the notion that confinement effects are of minor importance for the two-stage assembly process in levitating drops. Assembly in levitating droplets that are influenced by surface tension typically result in a dried bead with a coreshell type structure (Figure S4), which was not observed in the current study.

We have complemented the time-resolved SAXS study and followed the later stage of the field-directed assembly process using light microscopy (Figure 4). Snapshots of a single region of the assembly at $6 \mathrm{mT}$ confirmed that the primary mesocrystals (Figure 4a) assemble to form elongated structures (Figure 4b) that subsequently attach to each other to form long fibers that are aligned with respect to the magnetic field (Figures $4 c$ and $4 d$ ). The snapshots in Figure 4 represent a time-series of one experiment and the highlighted mesocrystal fragments of increasing sizes in Figures $4 a-c$ assemble and generate the fiber shown in Figure $4 \mathrm{~d}$. Coarsening of the fibers through lateral growth was observed at longer times (Figure 4e). Video S1 shows that two mesocrystals begin to approach already at separation distances of about $8-10 \mu \mathrm{m}$. The fiber width and width distribution decrease with increasing magnetic field strength and with decreasing initial nanoparticle concentration (Figure S5). Although fiber length was difficult to measure because of fractures in the fibers caused by capillary pressure during drying, it was possible to find fibers with lengths of $1 \mathrm{~mm}$, which were assembled at $6 \mathrm{mT}$ and a relatively high initial concentration $\left(5 \mathrm{mg} \mathrm{mL}^{-1}\right)$. Fibers formed from dispersions with a low initial concentration $\left(0.5 \mathrm{mg} \mathrm{mL}^{-1}\right)$ were relatively short and sometimes branched (Figure S5a-c). ${ }^{34}$ The decrease in fiber width with increasing magnetic field strength indicates that oriented attachment occurs for smaller mesocrystal sizes compared to low field strengths. The number of nanocubes in the mesocrystalline fibers can be estimated by assuming a square-shaped cross section of the fiber and a simple cubic packing of the nanocubes with an edge length of 

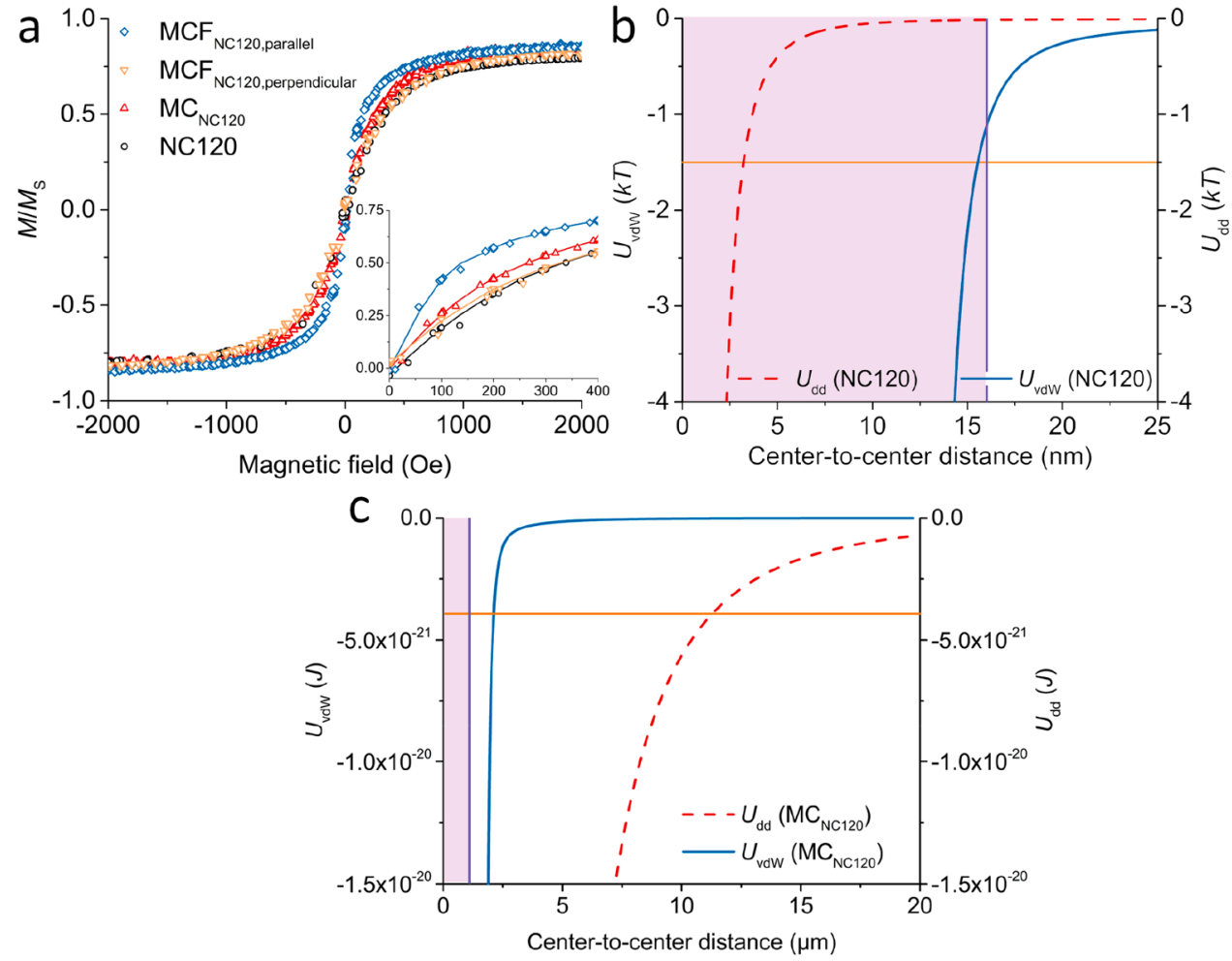

Figure 5. Magnetic properties and interaction energies. (a) Normalized magnetization $M / M_{\mathrm{S}}$ vs applied field curves for a diluted NC120 dispersion in paraffin wax (black circles), $\mathrm{MC}_{\mathrm{NC120}}$ grown on a substrate (red triangles up), and $\mathrm{MCF}_{\mathrm{NC} 120}$ grown on a substrate and measured parallel (blue diamonds) and perpendicular (orange triangles down) to the long axis of the fiber (blue diamonds). The inset displays the normalized magnetization at low magnetic fields. The solid lines are guides for the eye. (b) Dipolar interaction energy $U_{\text {dd }}$ between head-to-tail aligned magnetic moments of two NC120 (red dashed line), and van der Waals interaction energy $U_{\text {vdw }}$ of two face-to-face oriented NC120 (blue solid line). The horizontal orange line is the thermal energy, $3 / 2 k T$. The purple line and marked area indicates the distance of closest approach. (c) Dipolar interaction energy $U_{\mathrm{dd}}$ between two mesocrystals $\mathrm{MC}_{\mathrm{NC} 120}$ consisting of $100 \times 100 \times 100$ nanocubes and vdW interaction energy between face-to-face oriented $\mathrm{MC}_{\mathrm{NC} 120}$.

$12 \mathrm{~nm}$ and a $2 \mathrm{~nm}$ thick oleic acid capping layer. Considering a typical fiber length of $100 \mu \mathrm{m}$ and that the cross-section areas ranges from $80 \times 80$ to $700 \times 700$ nanocubes, each fiber is estimated to contain between $5 \times 10^{5}$ to $3 \times 10^{9}$ nanocubes depending on the assembly conditions. The cuboidal shape of the "primary" mesocrystals can be extracted from SEM images of mesocrystals grown in the absence of a magnetic field in both a levitating droplet and on a substrate (Figure S6).

Vibrating sample magnetometer (VSM) measurements (Figure 5a) showed that the mesocrystalline fibers $\left(\mathrm{MCF}_{\mathrm{NC120}}\right)$ grown in the presence of a field $(6 \mathrm{mT})$ had a larger saturation magnetization along $\left(189 \mathrm{kA} \mathrm{m}^{-1}\right)$ than perpendicular $\left(181 \mathrm{kA} \mathrm{m}^{-1}\right)$ to the long axis of the fibers (Figure 5a). This difference in saturation magnetization is line with previous reports and caused by dipolar couplings within a fiber but also between adjacent fibers. ${ }^{12,18}$ The saturation magnetization for small cuboidal mesocrystals $\left(\mathrm{MC}_{\mathrm{NC120}}\right)$ grown in the absence of a magnetic field $\left(123 \mathrm{kA} \mathrm{m}^{-1}\right)$ was much smaller than that of an individual NC120 $\left(257 \mathrm{kA} \mathrm{m}^{-1}\right)$, which could be caused by antiferromagnetic coupling in larger assemblies. ${ }^{35}$ The saturation magnetization for NC120 was similar to previously reported values for iron oxide nanoparticles. Both the single nanoparticles as well as the assembled structures displayed very low coercivities. Micromagnetic measurements on single mesocrystals are needed to elucidate why the assembled structures retained the superparamagnetic behavior of the nanocubes.
The field-induced alignment of the magnetic moments was estimated from the Zeeman energy. ${ }^{8,35}$ The estimated Zeeman energy of NC120 at full magnetization, $U_{\mathrm{Zeeman}, \mathrm{NC} 120}=m \mu_{0} \mathrm{H} \approx$ $2.6 \times 10^{-21} \mathrm{~J}$, where $m$ is the magnetic moment of a single nanoparticle and $\mu_{0} H=0.006 \mathrm{~T}$ the applied magnetic field, was smaller than the thermal energy $3 / 2 k T\left(\approx 6.2 \times 10^{-21} \mathrm{~J}, k\right.$ Boltzmann constant, temperature $T=298 \mathrm{~K})$, which suggests that the weak field $(6 \mathrm{mT})$ did not align the magnetic moments within the nanocubes. The Zeeman energy for a cuboidal mesocrystal with an edge length of $1.5-1.7 \mu \mathrm{m}$ can at a first approximation be estimated from the macro-spin approximation, yielding $U_{\text {Zeeman,MCNC120 }} \approx 1.3 \times 10^{-15} \mathrm{~J}$, which is several orders of magnitude larger than the thermal energy.

The magnetic moments $m=\mu_{0} M V$, with $\mu_{0}, M$, and $V$ being the vacuum permeability, the magnetization per unit volume, and the volume of a single particle, respectively, of NC120 $\left(\sim 26 \mathrm{kA} \mathrm{m}^{-1}\right)$ and $\mathrm{MC}_{\mathrm{NC} 120}\left(\sim 9 \mathrm{kA} \mathrm{m}^{-1}\right)$ at $6 \mathrm{mT}$, were extracted from the $\mathrm{M}-\mathrm{H}$ curves and used to calculate the dipolar interaction energy $U_{\mathrm{dd}}$ between two magnetic dipoles using

$$
U_{\mathrm{dd}}=\frac{m^{2}}{4 \pi \mu_{0} r^{3}}\left(1-3 \cos ^{2} \theta\right)
$$

The angle $\theta$ corresponds to the polar angle in a spherical coordinate system between two magnetic moments, separated by a center-to-center distance $r$, with respect to the applied 
magnetic field. Equation 2 assumes that the magnetic moments for the interacting particles have the same magnitude and are aligned with respect to the field.

The vdW interaction energy of two face-to-face oriented nanocubes with edge length $l$ separated by a surface-to-surface distance $d$ can be calculated by

$$
\begin{aligned}
& U_{\mathrm{vdW}, \text { cubes }}=-\left.\frac{A_{\text {Hamaker }}}{\pi^{2}} K_{f}(x)\right|_{d+l, d+l} ^{d+2 l, d} \\
& \left.K_{f}(x)\right|_{d+l, d+l} ^{d+2 l, d}=K_{f}(d+2 l)-2 K_{f}(d+l)+K_{f}(d) \\
& K_{f}(x)=\frac{1}{4} \ln \left(\frac{x^{4}+2 x^{2} l^{2}+l^{4}}{x^{4}+2 x^{2} l^{2}}\right)+2\left(\frac{x^{2}-l^{2}}{4 l x}\right) \tan ^{-1}\left(\frac{l}{x}\right) \\
& +\frac{x\left(2 l^{2}\right)^{3 / 2}}{6 l^{4}} \tan ^{-1}\left[\frac{x}{\left(2 l^{2}\right)^{1 / 2}}\right]+2 l\left(\frac{1}{6 x^{2}}+\frac{1}{6 l^{2}}\right) \\
& \quad \times\left(x^{2}+l^{2}\right)^{1 / 2} \tan ^{-1}\left(\frac{l}{\left(x^{2}+l^{2}\right)^{1 / 2}}\right)
\end{aligned}
$$

We have used a Hamaker constant $A_{\text {Hamaker }}=21 \mathrm{zJ}$ for magnetite in decane. ${ }^{36}$ The dipolar interaction energy between face-to-face oriented nanocubes and head-to-tail aligned magnetic moments, respectively, at a field strength of $6 \mathrm{mT}$ was negligible compared to the $\mathrm{vdW}$ attraction (Figure $5 \mathrm{~b}$ ). This supports that the assembly of nanocubes into the primary cuboidal mesocrystals is driven by vdW interactions as the particle concentration increases and that the influence of a weak magnetic field is insignificant.

The interactions between two mesocrystals consisting of 100 $\times 100 \times 100$ nanocubes, however, is dominated by the dipolar interactions, which are several orders of magnitude stronger and longer in range than the vdW attraction. Estimates based on the macro-spin model and an average angle $\langle\theta\rangle \approx 30^{\circ}$ (obtained from Video S1) suggests that the dipolar attraction becomes stronger than the thermal energy $3 / 2 k T$ already at a center-to-center distance of about $9.7 \mu \mathrm{m}$ (Figure 5c), which corresponds well to the approach distance of $10 \mu \mathrm{m}$ observed by optical microscopy (Video S1). Hence, magnetic dipolar interactions come into play for larger mesocrystals, whereas nanocube self-assembly is dominated by vdW interactions. The transition from vdW-driven growth of the primary cuboidal mesocrystals to magnetic-field-driven secondary assembly of the mesocrystals into fibers is expected to occur when the dipolar interactions between the growing cuboidal mesocrystals become sufficiently large and the separation distance between adjacent mesocrystals sufficiently small. The dipolar interaction energy in weak magnetic fields, where the magnetic moments within each nanocube is only partially aligned, is expected to be lower at lower magnetic field strength, which suggest that the transition from $3 \mathrm{D}$ to $1 \mathrm{D}$ assembly should occur for smaller sizes of the cuboidal mesocrystals at higher magnetic field strengths. Indeed, we found that the diameter of the fibers depends on the initial particle concentration and magnetic field strength and increases from a diameter equivalent to 80 nanocubes $(1.3 \mu \mathrm{m})$ at $6 \mathrm{mT}$ to 700 nanocubes $(11 \mu \mathrm{m})$ at $1.5 \mathrm{mT}$ (Figure S5). The estimates of the vdW and dipolar interaction energies suggest that the size dependence of vdW and magnetic field-driven assembly can be used to tune the size and morphology of the anisotropic mesocrystals.
In summary, we have shown that the assembly in weak magnetic fields of oleate-capped superparamagnetic iron oxide nanocubes into mesocrystalline fibers with a high degree of positional order and crystallographic coherence occurs in two stages. The first stage formation and growth of micrometersized cuboidal mesocrystals is initiated when the particle concentration becomes sufficiently high and was shown to be unaffected by the weak magnetic field. The transition from 3D growth of the primary cuboidal mesocrystals to the second stage $1 \mathrm{D}$ assembly of the elongated fibers depends on the initial particle concentration and the strength of the magnetic field. The increase of the width of the fibers from a diameter equivalent to 80 nanocubes $(1.3 \mu \mathrm{m})$ at $6 \mathrm{mT}$ to 700 nanocubes $(11 \mu \mathrm{m})$ at $1.5 \mathrm{mT}$ was related to the size and field dependence of the isotropic van der Waals and directional dipolar interactions. Controlled assembly of mesocrystalline fibers with tunable diameters could have potential applications for fabrication of spintronic components and for hyperthermia cancer treatment. $^{8,37}$

\section{ASSOCIATED CONTENT}

\section{Supporting Information}

The Supporting Information is available free of charge at https://pubs.acs.org/doi/10.1021/acs.nanolett.0c02770.

Synthesis of magnetite nanocubes, characterization methods, time-dependent concentration and scattering invariant, tunable assembly of mesocrystalline fibers in dependence of applied magnetic field (PDF)

Video S1 (AVI)

\section{AUTHOR INFORMATION}

\section{Corresponding Authors}

Lennart Bergström - Department of Materials and Environmental Chemistry, Stockholm University, 10691 Stockholm, Sweden; 이이.org/0000-0002-5702-0681; Email: lennart.bergstrom@mmk.su.se

Martin Kapuscinski - Department of Materials and Environmental Chemistry, Stockholm University, 10691 Stockholm, Sweden; 이이이.org/0000-0001-5435-2958; Email: martin.kapuscinski@mmk.su.se

\section{Authors}

Pierre Munier - Department of Materials and Environmental Chemistry, Stockholm University, 10691 Stockholm, Sweden; (1) orcid.org/0000-0001-7402-0088

Mo Segad - Department of Materials and Environmental Chemistry, Stockholm University, 10691 Stockholm, Sweden

Complete contact information is available at:

https://pubs.acs.org/10.1021/acs.nanolett.0c02770

\section{Author Contributions}

M.K. and L.B. conceived the study. M.K. planned and designed the experiments. M.K., P.M., and M.S. acquired the SAXS data. M.K. performed the SAXS and magnetic data analysis and interpreted the data. M.K. and L.B. wrote the manuscript. All authors commented on the manuscript.

\section{Notes}

The authors declare no competing financial interest.

\section{ACKNOWLEDGMENTS}

We acknowledge the Swedish Research Council (V.R., grant no. 2019-05624) for funding this study. We are grateful for the 
insightful suggestions and discussions with A. Petukhov. We thank T. Church for linguistic revision. We would like to thank J. Paulin and P. Jansson for technical assistance with the acoustic levitator, G. Salazar-Alvarez for scientific discussions, V. Smetana for assistance in magnetic measurements, and T. S. Plivelic for providing the acoustic levitator. We are grateful to Tufvassons AB (Sigtuna, Sweden) for providing material to build the electromagnet. We acknowledge DESY (Hamburg, Germany), a member of the Helmholtz Association HGF, for the provision of experimental facilities, for the allocated beamtime (proposal ID: I-20180345 EC), and for the EU Travel Expense Grant (CALIPSOplus). Parts of this research were carried out at PETRA III, and we would like to thank Wiebke Ohm for assistance in using the P03 beamline. The research leading to this result has been supported by the project CALIPSOplus under the grant agreement 730872 from the EU Framework Programme for Research and Innovation HORIZON 2020.

\section{REFERENCES}

(1) Zhang, P.; Tachikawa, T.; Fujitsuka, M.; Majima, T. The Development of Functional Mesocrystals for Energy Harvesting, Storage, and Conversion. Chem. - Eur. J. 2018, 24, 6295-6307.

(2) Bergström, L.; Sturm (née Rosseeva), E. V.; Salazar-Alvarez, G.; Cölfen, H. Mesocrystals in Biominerals and Colloidal Arrays. Acc. Chem. Res. 2015, 48, 1391-1402.

(3) Ma, M.-G.; Cölfen, H. Mesocrystals - Applications and Potential. Curr. Opin. Colloid Interface Sci. 2014, 19, 56-65.

(4) Martinez-Boubeta, C.; Simeonidis, K.; Makridis, A.; Angelakeris, M.; Iglesias, O.; Guardia, P.; Cabot, A.; Yedra, L.; Estradé, S.; Peiró, F.; Saghi, Z.; Midgley, P. A.; Conde-Leborán, I.; Serantes, D.; Baldomir, D. Learning from Nature to Improve the Heat Generation of Iron-Oxide Nanoparticles for Magnetic Hyperthermia Applications. Sci. Rep. 2013, 3, 1652.

(5) Corr, S. A.; Byrne, S. J.; Tekoriute, R.; Meledandri, C. J.; Brougham, D. F.; Lynch, M.; Kerskens, C.; O’Dwyer, L.; Gun'ko, Y. K. Linear Assemblies of Magnetic Nanoparticles as MRI Contrast Agents. J. Am. Chem. Soc. 2008, 130, 4214-4215.

(6) Peiris, P. M.; Toy, R.; Doolittle, E.; Pansky, J.; Abramowski, A.; Tam, M.; Vicente, P.; Tran, E.; Hayden, E.; Camann, A.; Mayer, A.; Erokwu, B. O.; Berman, Z.; Wilson, D.; Baskaran, H.; Flask, C. A.; Keri, R. A.; Karathanasis, E. Imaging Metastasis Using an IntegrinTargeting Chain-Shaped Nanoparticle. ACS Nano 2012, 6, 87838795.

(7) Talapin, D. V.; Lee, J.-S.; Kovalenko, M. V.; Shevchenko, E. V. Prospects of Colloidal Nanocrystals for Electronic and Optoelectronic Applications. Chem. Rev. 2010, 110, 389-458.

(8) Wang, H.; Yu, Y.; Sun, Y.; Chen, Q. Magnetic Nanochains: A Review. Nano 2011, 6, 1-17.

(9) Zhang, S.; Pelligra, C. I.; Feng, X.; Osuji, C. O. Directed Assembly of Hybrid Nanomaterials and Nanocomposites. Adv. Mater. 2018, 30, 1705794.

(10) Dincer, I.; Tozkoparan, O.; German, S. V.; Markin, A. V.; Yildirim, O.; Khomutov, G. B.; Gorin, D. A.; Venig, S. B.; Elerman, Y. Effect of the Number of Iron Oxide Nanoparticle Layers on the Magnetic Properties of Nanocomposite LbL Assemblies. J. Magn. Magn. Mater. 2012, 324, 2958-2963.

(11) Grzelczak, M.; Vermant, J.; Furst, E. M.; Liz-Marzán, L. M. Directed Self-Assembly of Nanoparticles. ACS Nano 2010, 4, 35913605.

(12) Toulemon, D.; Rastei, M. V.; Schmool, D.; Garitaonandia, J. S.; Lezama, L.; Cattoën, X.; Bégin-Colin, S.; Pichon, B. P. Enhanced Collective Magnetic Properties Induced by the Controlled Assembly of Iron Oxide Nanoparticles in Chains. Adv. Funct. Mater. 2016, 26, $2454-2462$
(13) Sahoo, Y.; Cheon, M.; Wang, S.; Luo, H.; Furlani, E. P.; Prasad, P. N. Field-Directed Self-Assembly of Magnetic Nanoparticles. J. Phys. Chem. B 2004, 108, 3380-3383.

(14) Nakata, K.; Hu, Y.; Uzun, O.; Bakr, O.; Stellacci, F. Chains of Superparamagnetic Nanoparticles. Adv. Mater. 2008, 20, 4294-4299.

(15) Myrovali, E.; Maniotis, N.; Makridis, A.; Terzopoulou, A.; Ntomprougkidis, V.; Simeonidis, K.; Sakellari, D.; Kalogirou, O.; Samaras, T.; Salikhov, R.; Spasova, M.; Farle, M.; Wiedwald, U.; Angelakeris, M. Arrangement at the Nanoscale: Effect on Magnetic Particle Hyperthermia. Sci. Rep. 2016, 6, 37934.

(16) Wetterskog, E.; Klapper, A.; Disch, S.; Josten, E.; Hermann, R. P.; Rücker, U.; Brückel, T.; Bergström, L.; Salazar-Alvarez, G. Tuning the Structure and Habit of Iron Oxide Mesocrystals. Nanoscale 2016, $8,15571-15580$

(17) Singh, G.; Chan, H.; Udayabhaskararao, T.; Gelman, E.; Peddis, D.; Baskin, A.; Leitus, G.; Král, P.; Klajn, R. Magnetic Field-Induced Self-Assembly of Iron Oxide Nanocubes. Faraday Discuss. 2015, 181, 403-421.

(18) Jiang, C.; Leung, C. W.; Pong, P. W. T. Magnetic-Field-Assisted Assembly of Anisotropic Superstructures by Iron Oxide Nanoparticles and Their Enhanced Magnetism. Nanoscale Res. Lett. 2016, 11, 189.

(19) Singh, G.; Chan, H.; Baskin, A.; Gelman, E.; Repnin, N.; Král, P.; Klajn, R. Self-Assembly of Magnetite Nanocubes into Helical Superstructures. Science 2014, 345, 1149-1153.

(20) Fu, Z.; Xiao, Y.; Feoktystov, A.; Pipich, V.; Appavou, M. S.; Su, Y.; Feng, E.; Jin, W.; Brückel, T. Field-Induced Self-Assembly of Iron Oxide Nanoparticles Investigated Using Small-Angle Neutron Scattering. Nanoscale 2016, 8, 18541-18550.

(21) Kralj, S.; Makovec, D. Magnetic Assembly of Superparamagnetic Iron Oxide Nanoparticle Clusters into Nanochains and Nanobundles. ACS Nano 2015, 9, 9700-9707.

(22) Jia, B.; Gao, L. Morphological Transformation of Fe3O4 Spherical Aggregates from Solid to Hollow and Their Self-Assembly under an External Magnetic Field. J. Phys. Chem. C 2008, 112, 666671.

(23) Rastei, M. V.; Pierron-Bohnes, V.; Toulemon, D.; Bouillet, C.; Kákay, A.; Hertel, R.; Tetsi, E.; Begin-Colin, S.; Pichon, B. P. DefectDriven Magnetization Configuration of Isolated Linear Assemblies of Iron Oxide Nanoparticles. Adv. Funct. Mater. 2019, 29, 1903927.

(24) Faure, B.; Salazar-Alvarez, G.; Bergström, L. Hamaker Constants of Iron Oxide Nanoparticles. Langmuir 2011, 27, 86598664.

(25) Kumar, J. S.; Paul, P. S.; Raghunathan, G.; Alex, D. G. A Review of Challenges and Solutions in the Preparation and Use of Magnetorheological Fluids. Int. J. Mech. Mater. Eng. 2019, 14, 13.

(26) Brune, H. Assembly and Probing of Spin Chains of Finite Size. Science 2006, 312, 1005-1006.

(27) Kapuscinski, M.; Agthe, M.; Lv, Z. P.; Liu, Y.; Segad, M.; Bergström, L. Temporal Evolution of Superlattice Contraction and Defect-Induced Strain Anisotropy in Mesocrystals during Nanocube Self-Assembly. ACS Nano 2020, 14, 5337-5347.

(28) Lv, Z.-P.; Kapuscinski, M.; Bergström, L. Tunable Assembly of Truncated Nanocubes by Evaporation-Driven Poor-Solvent Enrichment. Nat. Commun. 2019, 10, 4228.

(29) Agthe, M.; Plivelic, T. S.; Labrador, A.; Bergström, L.; SalazarAlvarez, G. Following in Real Time the Two-Step Assembly of Nanoparticles into Mesocrystals in Levitating Drops. Nano Lett. 2016, 16, 6838-6843.

(30) Reich, S.; Letzel, A.; Menzel, A.; Kretzschmar, N.; Gökce, B.; Barcikowski, S.; Plech, A. Early Appearance of Crystalline Nanoparticles in Pulsed Laser Ablation in Liquids Dynamics. Nanoscale 2019, 11, 6962-6969.

(31) van Heugten, A. J. P.; Landman, J.; Petukhov, A. V.; Vromans, H. Study of Petrolatum Structure: Explaining Its Variable Rheological Behavior. Int. J. Pharm. 2018, 540, 178-184.

(32) Abécassis, B.; Testard, F.; Spalla, O. Gold Nanoparticle Superlattice Crystallization Probed in Situ. Phys. Rev. Lett. 2008, 100, 115504. 
(33) Imai, H. Mesostructured Crystals: Growth Processes and Features. Prog. Cryst. Growth Charact. Mater. 2016, 62, 212-226.

(34) Tanase, M.; Silevitch, D. M.; Hultgren, A.; Bauer, L. A.; Searson, P. C.; Meyer, G. J.; Reich, D. H. Magnetic Trapping and SelfAssembly of Multicomponent Nanowires. J. Appl. Phys. 2002, 91, 8549-8551.

(35) Mehdizadeh Taheri, S.; Michaelis, M.; Friedrich, T.; Förster, B.; Drechsler, M.; Römer, F. M.; Bösecke, P.; Narayanan, T.; Weber, B.; Rehberg, I.; Rosenfeldt, S.; Förster, S. Self-Assembly of Smallest Magnetic Particles. Proc. Natl. Acad. Sci. U. S. A. 2015, 112, 1448414489.

(36) Lu, C.; Akey, A. J.; Dahlman, C. J.; Zhang, D.; Herman, I. P. Resolving the Growth of 3D Colloidal Nanoparticle Superlattices by Real-Time Small-Angle X-ray Scattering. J. Am. Chem. Soc. 2012, 134, 18732-18738.

(37) Jordan, A.; Scholz, R.; Wust, P.; Fähling, H.; Felix, R. Magnetic Fluid Hyperthermia (MFH): Cancer Treatment with AC Magnetic Field Induced Excitation of Biocompatible Superparamagnetic Nanoparticles. J. Magn. Magn. Mater. 1999, 201, 413-419. 